\title{
FT-IR Analysis of Urinary Stones: A Helpful Tool for Clinician Comparison with the Chemical Spot Test
}

\author{
Aniello Primiano, ${ }^{1}$ Silvia Persichilli, ${ }^{1}$ Giovanni Gambaro, ${ }^{2}$ \\ Pietro Manuel Ferraro, ${ }^{2}$ Alessandro D'Addessi, ${ }^{3}$ Andrea Cocci, ${ }^{1}$ Arcangelo Schiattarella, ${ }^{1}$ \\ Cecilia Zuppi, ${ }^{1}$ and Jacopo Gervasoni ${ }^{1}$ \\ ${ }^{1}$ Istituto di Biochimica e Biochimica Clinica, Università Cattolica del Sacro Cuore, Largo Agostino Gemelli 8, 00168 Rome, Italy \\ ${ }^{2}$ Divisione di Nefrologia Complesso Integrato Columbus, Dipartimento di Scienze Mediche, \\ Policlinico Gemelli Università Cattolica del Sacro Cuore, Largo Agostino Gemelli 8, 00168 Rome, Italy \\ ${ }^{3}$ Clinica Urologica, Policlinico Gemelli Università Cattolica del Sacro Cuore, Largo Agostino Gemelli 8, 00168 Rome, Italy
}

Correspondence should be addressed to Silvia Persichilli; spersichilli@rm.unicatt.it

Received 25 February 2014; Accepted 27 March 2014; Published 27 April 2014

Academic Editor: Vincent Sapin

Copyright ( 2014 Aniello Primiano et al. This is an open access article distributed under the Creative Commons Attribution License, which permits unrestricted use, distribution, and reproduction in any medium, provided the original work is properly cited.

Background. Kidney stones are a common illness with multifactorial etiopathogenesis. The determination of crystalline and molecular composition and the quantification of all stone components are important to establish the etiology of stones disease but it is often laborious to obtain using the chemical method. The aim of this paper is to compare chemical spot test with FT-IR spectroscopy, for a possible introduction in our laboratory. Methods. We analyzed 48 calculi using Urinary Calculi Analysis kit in accordance with the manufacturer's instructions. The same samples were analyzed by FT-IR using the Perkin Elmer Spectrum One FT-IR Spectrometer. All FT-IR spectra of kidney stones were then computer matched against a library of spectra to generate a report on the various components. Results. On the basis of FT-IR analysis, the 48 calculi were divided into three groups: pure stone, mixed stone, and pure stone with substances in trace. Results of each group were compared with those obtained with chemical spot test. A general disagreement between methods was observed. Conclusions. According to our data, the introduction of the FT-IR technique in clinical chemistry laboratory may be more responsive to clinician expectations.

\section{Introduction}

Nephrolithiasis is a common disease, occurring in both industrialized and developing countries and mainly affecting adults aged 20-60 years [1].

A recent survey in Italy has shown a prevalence of $7.5 \%$ in an urban population [2]. Stone formation is the end result of a multistep process in which the balance of factors that promote crystallization of urinary salts and factors that inhibit crystallization is perturbed. Urinary stones may be composed of calcium oxalate monohydrate (COM, whewellite), calcium oxalate dihydrate (COD, weddellite), carbonate apatite (CA, dahllite), ammonium urate, magnesium ammonium phosphate (PAM, Struvite), calcium hydrogen phosphate dihydrate (brushite), uric acid (AU0 anhydrous form and
AU2 dihydrate form, uricite) and its salts, cystine, xanthine, 2,8-dihydroxyadenine, and drugs [3].

It is worldwide underlined that the determination of crystalline and molecular composition and the quantification of all stone components are helpful to establish the etiology of stones disease. Different methodologies exist for the analysis of renal stones. These include qualitative "dry" chemical spot tests and quantitative X-ray crystallography, infrared spectroscopy (FT-IR), and "wet" chemistry tests [4].

Chemical spot tests are relatively inaccurate because of false-positive and false-negative results and do not allow distinguishing between the crystalline phases. Among physical methods, X-ray diffraction is appropriate for quantification of mineral samples, but it cannot adequately detect amorphous species such as carbapatite or struvite. FT-IR spectroscopy 
is the most appropriate technique for stones analysis and is becoming the gold standard for stone analysis $[3,5]$. The infrared spectrum originates from the vibrational motion of the molecules. The vibrational frequencies are a kind of fingerprint of the compounds. This property is used for characterization of organic and inorganic compounds present in renal calculi. The band intensities are proportional to the compound concentration and hence qualitative estimations are also obtained. FT-IR spectroscopy leads to unambiguous information about the stone composition, both for main substances and trace elements, all essentials to guide therapy [6].

The aim of this paper is to compare a semiquantitative method (DiaSys) with a quantitative method (FT-IR spectroscopy technique) for urinary stone analysis, in order to introduce in our laboratory a more reliable technique.

\section{Material and Methods}

We analyzed 48 urinary stones, from 48 patients (28 men and 20 women, age range 21-75) from our Divisions of Nephrology and Urology. Stones were analyzed by both spot test and FT-IR method as described below. Shape, colour, size and weight were registered for each stone at the time of delivery. The stones submitted to analysis were washed with deionized water and dried at room temperature for $24 \mathrm{~h}$; subsequently the stones were powdered in a mortar and aliquoted in 2 vials before being subjected to the following analysis.

2.1. Chemical Spot Test. Spot test analysis for the qualitative tests of urinary calculi composition was performed according to kit instructions (Urinary Calculi Analysis kit, DiaSys, Diagnostic System GmbH, Holzheim, Germany). This method allows detecting the presence of cystine and following ions usually present in urinary calculi: carbonate, calcium, oxalate, ammonium, phosphate, magnesium, and urate. The assay consists of the addition of chemical reagents labeled R1 to R15 dropwise to the finely pulverized sample and placed into a vessel with $50 \mathrm{~mL}$ of distilled water. Then the appearance of certain colors, precipitates, or air bubbles would indicate positive results for one of the ions and cystine [7]. For example, for the phosphate, while shaking, five drops of reagent 9 (ammonium molybdate solution) and five drops of reagent 10 (4-methyl-aminophenol sulfate, sodium bisulfite) were added in reaction vessel; after five-minute incubation the newly appearing colour was matched with the kit colour scale for the semiquantitative analysis.

2.2. FT-IR Analysis. The second aliquot of the pulverized stone was mixed with an inert powdered support (dried potassium bromide) in a proportion of 0.5 to $2 \%$ in agate mortar. This mixture was transferred into an appropriate die and pressed at $10 \mathrm{t} / \mathrm{cm}^{2}$ to form a transparent pellet $13 \mathrm{~mm}$ in diameter. The pellet assembled in a holder was placed in the IR beam of the spectrometer. The spectral region investigated was from 4000 to $400 \mathrm{~cm}^{-1} ; 32$ scans were averaged with a $4 \mathrm{~cm}^{-1}$ resolution for each spectrum. A background spectrum was collected before every analysis, for the sample blank.
Again a background spectrum was measured to provide a relative scale for the absorption intensity. Background spectra were performed at air or pure $\mathrm{KBr}$ pellet. Spectra were recorded by means of a Perkin Elmer Spectrum One [8].

Spectra were then computer-matched with the Euclidean search application, a tool of SPECTRA NICODOM IR Library (obtained from Nicodom s.r.o., Hlavni 2727 CZ-14100 Praha 4, Czech Republic, EU) that compares the unknown spectrum with reference spectra contained in the library between 4000 and $400 \mathrm{~cm}^{-1}$. A report is then generated for the various stone components. The results of the automatic comparison for a spectrum identification were provided as a list of the best-fitting spectra with their score. The score value can range from 0.000 to 1.000 . Score 1.000 indicates a perfect likeness between the unknown spectrum and the reference one. In each case, a visual inspection of the spectra was performed to check the results.

2.3. Method Comparison. On the basis of FT-IR analysis, the 48 calculi were divided into three groups: pure stone $(n=$ $23)$, mixed stone $(n=19)$, and pure stone with substances in trace $(n=6)$. Results of each group were compared with those obtained with chemical spot test.

Classification criteria were established by comparing results obtained with the two methods in reference to the identification of cystine and the ionic species as follows:

(i) agreement: when FT-IR and the chemical spot test identify the same components,

(ii) partial agreement: when the chemical spot test identifies the main component detected by FT-IR in addition to other ions not attributable to a particular crystalline species,

(iii) disagreement: when the spot test does not identify the main substance or when it identifies the main component but also other ions referable to particular crystalline species which were not detected by FT-IR.

\section{Results}

The percentage values of major constituents for the chemical spot test and FT-IR method are shown in Tables 1(a) and 1(b), respectively. As clearly shown the major components detected by both techniques were calcium and oxalate. However, the two tests differ remarkably in the detection of oxalate, magnesium, ammonium, and cystine (a higher detection yield by the chemical spot test) and in the detection of urate and carbonate (a lower detection yield by the chemical spot test) (Table 2).

In the pure stone group (Table 3 ) a partial disagreement between the two methods was shown. In fact, they were in agreement only in $11 / 23$ cases $(47.8 \%)$, in partial agreement in $4 / 23$ (17.4\%), and in disagreement in $8 / 23$ (34.8\%) cases.

Tables 4 and 5 show that also results relative to mixed stone and pure stone with substances in trace groups were characterized by a general disagreement. In fact for the mixed stone group the two methods were in agreement only in 
TABLE 1: Chemical constituents of urinary stone (\%) obtained using chemical spot test (a) and FT-IR analysis (b).

(a) Chemical spot test analysis

\begin{tabular}{lcc}
\hline Components detected & Number & Frequency (\%) \\
\hline Oxalate & $45 / 48$ & 93.7 \\
Calcium & $39 / 48$ & 81.2 \\
Magnesium & $16 / 48$ & 33.3 \\
Phosphate & $14 / 48$ & 29.1 \\
Urate & $9 / 48$ & 18.7 \\
Ammonium & $7 / 48$ & 14.5 \\
Cystine & $5 / 48$ & 10.4 \\
Carbonate & $3 / 48$ & 6.2 \\
\hline
\end{tabular}

(b) FT-IR analysis

\begin{tabular}{lcc}
\hline Components detected & Number & Frequency (\%) \\
\hline Calcium oxalate monohydrate & $32 / 48$ & 66.6 \\
Carbonate apatite & $16 / 48$ & 33.3 \\
Anhydrous uric acid & $12 / 48$ & 25.0 \\
Calcium oxalate dihydrate & $8 / 48$ & 16.6 \\
Dihydrate uric acid & $2 / 48$ & 4.1 \\
Magnesium ammonium phosphate & $2 / 48$ & 4.1 \\
Cystine & $2 / 48$ & 4.1 \\
Atazanavir & $1 / 48$ & 1.0 \\
\hline
\end{tabular}

TABLe 2: Comparison between urinary stone composition (frequency \%) obtained with chemical spot test (extrapolated associating the single chemical constituents) and FT-IR analysis.

\begin{tabular}{|c|c|c|}
\hline \multicolumn{3}{|c|}{ Chemical spot test versus FT-IR in components identification } \\
\hline $\begin{array}{l}\text { Components } \\
\text { detected }\end{array}$ & $\begin{array}{l}\text { Frequency (\%) by chemical } \\
\text { spot test }\end{array}$ & $\begin{array}{c}\text { Frequency }(\%) \\
\text { by FT-IR }\end{array}$ \\
\hline Oxalate & 93.7 & 75.0 \\
\hline Calcium & 81.2 & 77.1 \\
\hline Magnesium & 33.3 & 4.1 \\
\hline Phosphate & 29.1 & 37.5 \\
\hline Urate & 18.7 & 25.0 \\
\hline Ammonium & 14.5 & 4.1 \\
\hline Cystine & 10.4 & 4.1 \\
\hline Carbonate & 6.2 & 37.5 \\
\hline
\end{tabular}

$3 / 19$ (15.8\%), in partial agreement in $4 / 19(21.0 \%)$, and in disagreement in 12/19 (63.2\%) cases.

For the pure stone with substances in trace group the two methods were in agreement in none $(0 \%)$, in partial agreement in 5/6 (83.3\%), and in disagreement in 1/6 (16.7\%) cases.

Thus, the mixed stone groups show the major disagreement.

\section{Discussion}

Urolithiasis is a frequent disease whose incidence is progressively increased in the last years in both men and women.
TABLE 3: Agreement between results obtained with chemical spot test and FT-IR in the pure stone group.

\begin{tabular}{lccc}
\hline \multicolumn{4}{c}{ Pure stones } \\
$\begin{array}{l}\text { Substances detected } \\
(n)\end{array}$ & $\begin{array}{c}\text { Agreement } \\
(n)\end{array}$ & $\begin{array}{c}\text { Partial } \\
\text { agreement } \\
(n)\end{array}$ & $\begin{array}{c}\text { Disagreement } \\
(n)\end{array}$ \\
\hline Calcium oxalate & 10 & 3 & 1 \\
monohydrate (14) & 0 & 0 & 6 \\
Anhydrous uric acid (6) & 1 & 1 & 0 \\
Cystine (2) & 0 & 0 & 1 \\
Atazanavir (1) & $11(47.8 \%)$ & $4(17.4 \%)$ & $8(34.8 \%)$ \\
\hline Total (23) & & &
\end{tabular}

Urinary stone composition is important both for correct diagnosis and for patient follow-up. Among the methods available for urinary stone analysis, chemical analysis has been traditionally used most widely due to its ease and low cost even if this technique is time consuming and necessitates large stone samples.

Guidelines on Urolithiasis of European Association of Urology 2013 underlines the obsolescence of chemical analysis and recommends the use of FT-IR for urinary stone analysis [3]. As reported by some authors, chemical methods presented an error rate from 6.5 to $94 \%$ confirming the dramatic inaccuracy of these methods [6]. The reason for such an inertia in the implementation in many laboratories of the FTIR method while continuing to use qualitative chemical tests most likely resides in the uncertainty on whether an accurate characterization of the stone composition is really useful for the metaphylaxis of nephrolithiasis [9]. No doubt that in very rare forms whose recognition can be only obtained with the FT-IR method (drugs or xanthine or 2,8-dihydroxyadenine stones) this is crucial for the rational treatment of the patient. Actually in this study the spot test could not detect the atazanavir in a stone which was incorrectly recognized as composed by oxalate, a wrong diagnosis which could have led to an incorrect medical treatment. However, these are really very rare cases. Hence, especially for the calcium containing stones still some believe that stone analysis could not be useful in the investigation of renal stone patients [9].

Yet, we think that there is one stronger reason to discourage the use of chemical spot tests, that is, the many relevant drawbacks of these techniques even for the very frequent forms of nephrolithiasis.

In this study, 48 urinary stones were analyzed using both the chemical spot test and the FT-IR method and results were compared. As expected, the most common components detected by both methods were calcium and oxalate. However we observed oxalate in $75.0 \%$ and calcium in $77.1 \%$ using the FT-IR method, while we observed oxalate in $93,7 \%$ and calcium in $81.2 \%$ using the chemical spot test. The difference between the two methods in detecting oxalate is remarkable since it was incorrectly recognized in $25.0 \%$ more stones.

However, the insufficient discrimination by the chemical spot method of uric acid stones is even more amazing. In fact, the chemical method does not or, only partially, recognize the 
TABle 4: Agreement between results obtained with chemical spot test and FT-IR in the pure stone with substances in trace group.

Pure stones with substance in trace

Components detected $(n)$

Agreement $(n)$

Calcium oxalate monohydrate + carbonate apatite trace (4)

Calcium oxalate dihydrate + carbonate apatite trace (1)

Calcium oxalate monohydrate + calcium oxalate dihydrate trace (1)

Total (6)

\begin{tabular}{ccc}
0 & Partial agreement $(n)$ & Disagreement $(n)$ \\
0 & 3 & 1 \\
0 & 1 & 0 \\
$0(0.0 \%)$ & 1 & 0 \\
\hline
\end{tabular}

TABLE 5: Agreement between results obtained with chemical spot test and FT-IR in the mixed stone group.

\begin{tabular}{|c|c|c|c|}
\hline \multicolumn{4}{|l|}{ Mixed stones } \\
\hline Substances detected $(n)$ & $\begin{array}{c}\text { Agreement } \\
(n)\end{array}$ & $\begin{array}{c}\text { Partial } \\
\text { agreement }(n)\end{array}$ & $\begin{array}{c}\text { Disagreement } \\
(n)\end{array}$ \\
\hline Anhydrous uric acid + calcium oxalate monohydrate (3) & 2 & 0 & 1 \\
\hline Calcium oxalate monohydrate + calcium oxalate dihydrate + carbonate apatite (4) & 0 & 2 & 2 \\
\hline Magnesium ammonium phosphate + carbonate apatite + calcium oxalate monohydrate $(1)$ & 0 & 0 & 1 \\
\hline Carbonate apatite + magnesium ammonium phosphate + protein $(1)$ & 0 & 0 & 1 \\
\hline Anhydrous uric acid + calcium oxalate monohydrate + carbonate apatite (1) & 0 & 0 & 1 \\
\hline Anhydrous uric acid + dihydrate uric acid (2) & 1 & 0 & 1 \\
\hline Carbonate apatite + calcium oxalate monohydrate + calcium oxalate dihydrate $(3)$ & 0 & 0 & 3 \\
\hline Calcium oxalate monohydrate + carbonate apatite (2) & 0 & 1 & 1 \\
\hline Calcium oxalate monohydrate + calcium oxalate dihydrate (1) & 0 & 1 & 0 \\
\hline Calcium oxalate dihydrate + carbonate apatite + protein $(1)$ & 0 & 0 & 1 \\
\hline Total (19) & $3(15.8 \%)$ & $4(21.0 \%)$ & $12(63.2 \%)$ \\
\hline
\end{tabular}

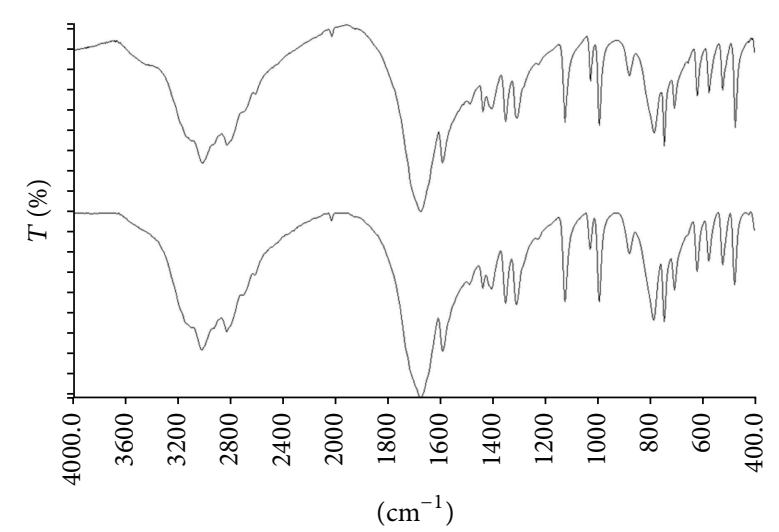

FIGURE 1: FT-IR spectrum of a uric acid anhydrous stone (top) matched with reference spectra contained in the NICODOM library. The reference spectrum of uric acid anhydrous stone with the best fit value was showed in the bottom.

presence of uric acid in stone samples (number 6) detected by FT-IR analysis (Figure 1). Since uric acid stones represent a significant percentage of urinary stones and deserve a specific medical treatment, a correct diagnosis of uric acid stones is crucial [10].

Also critical is the number of false positive ammonium or magnesium containing stones by the chemical spot method. In fact this finding may suggest to clinicians (despite absence of phosphates) that the stone is constituted by PAM, which mistakenly would lead to the recognition of an infective pathogenesis. Similarly, with this method five cystine containing stones were detected while the true number was two; this finding would lead to specific diagnostic and therapeutic measures which may even worsen the renal stone disease.

Another example can be found in calcium phosphates, which constitute a very heterogeneous group with multiple etiology including infections (in the case of carbonated calcium phosphate and whitlockite), hypercalciuric mechanism (in the case of brushite and octacalcium phosphate), and disorders related to tubular acidification function (in the case of carbonate apatite).

Moreover, there are significant differences on identification of substances present in trace in mixed stones, in fact FTIR technique shows a high sensitivity and allows an accurate identification of stone composition.

The elaborated treatment of samples and the subjective interpretation of results are the major disadvantages of the spot test, adding a variability out of control to structural method limits. Chemical methods have repeatedly proved to be unreliable in numerous quality control programs, with error rates in identifying certain components above 90\% [11]. On the contrast the FT-IR does not show a significant variability because it provides an easier and more standardized sample preparation, and the spectrum interpretation is based on strong scientific principles. The interpretation of results is aided from the use of Nicodom library which however is not always sufficiently sensitive and specific to differentiate species with similar spectral pattern and to detect minor components. Therefore, a skilled operator interpretation remains necessary. 
For these reasons, according to these results, the introduction of the FT-IR technique in our clinical laboratory may be more responsive to clinicians' expectations.

\section{Abbreviations}

COM: Calcium oxalate monohydrate

COD: Calcium oxalate dihydrate

CA: Carbonate apatite

PAM: Magnesium ammonium phosphate

AU0: Uric acid anhydrous

AU2: Uric acid dihydrate

FT-IR: Fourier transform infrared spectroscopy.

\section{Conflict of Interests}

The authors declare that there is no conflict of interests regarding the publication of this paper.

\section{References}

[1] M. Daudon, J. C. Doré, P. Jungers, and B. Lacour, "Changes in stone composition according to age and gender of patients: a multivariate epidemiological approach," Urological Research, vol. 32, no. 3, pp. 241-247, 2004.

[2] E. Croppi, P. M. Ferraro, L. Taddei, and G. Gambaro, "Prevalence of renal stones in an Italian urban population: a general practice-based study," Urological Research, vol. 40, no. 5, pp. 517$522,2012$.

[3] C. Türk, T. Knoll, A. Petrik et al., Guidelines on Urolithiasis, European Association of Urology, Arnhem, The Netherlands, 2013.

[4] I. A. Hashim and T. H. Zawawi, "Wet versus dry chemical analysis of renal stones," Irish Journal of Medical Science, vol. 168, no. 2, pp. 114-118, 1999.

[5] L. Benramdane, M. Bouatia, M. O. B. Idrissi, and M. Draoui, "Infrared analysis of urinary stones, using a single reflection accessory and a KBr pellet transmission," Spectroscopy Letters, vol. 41, no. 2, pp. 72-80, 2008.

[6] I. Singh, "Renal geology (quantitative renal stone analysis) by "Fourier transform infrared spectroscopy", International Urology and Nephrology, vol. 40, no. 3, pp. 595-602, 2008.

[7] B. Shabsoug, "Qualitative analysis of kidney stones by one wet chemical method for patients in Al-Qasseem area in Kingdom of Saudi Arabia," Journal of the Chemical Society of Pakistan, vol. 25, no. 4, pp. 334-336, 2003.

[8] L. M. Estepa, P. Levillain, B. Lacour, and M. Daudon, "Infrared analysis of urinary stones: a trial of automated identification," Clinical Chemistry and Laboratory Medicine, vol. 37, no. 11-12, pp. 1043-1052, 1999.

[9] M. J. Henderson, "Stone analysis is not useful in the routine investigation of renal stone disease," Annals of Clinical Biochemistry, vol. 32, no. 2, pp. 109-111, 1995.

[10] B. Shekarriz and M. L. Stoller, "Uric acid nephrolithiasis: current concepts and controversies," The Journal of Urology, vol. 168, no. 4 I, pp. 1307-1314, 2002.

[11] S. Gràcia-Garcia, F. Millán-Rodríguezb, F. Rousaud-Barónb et al., "Why and how we must analyze urinary calculi," Actas Urológicas Españolas, vol. 35, no. 6, pp. 354-362, 2011. 


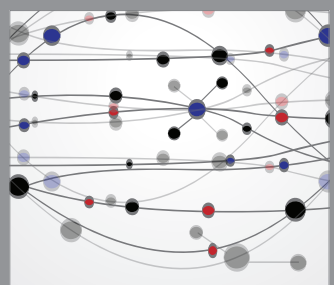

The Scientific World Journal
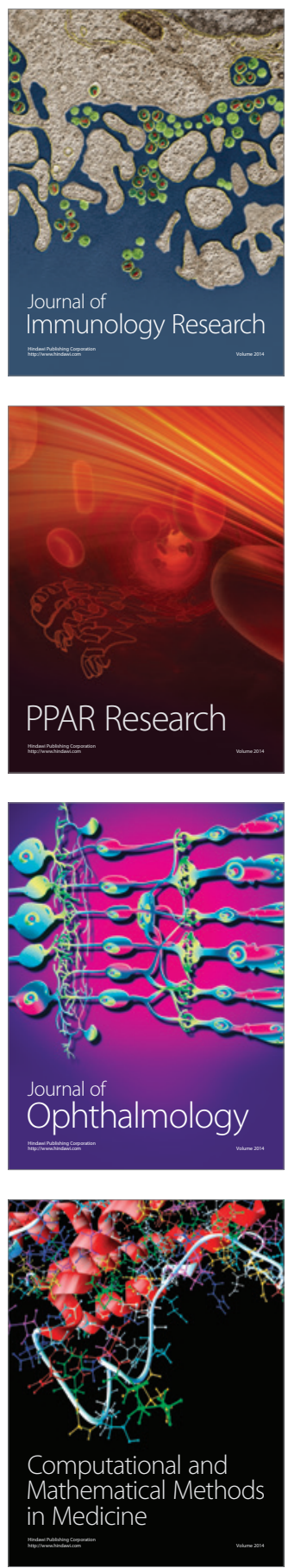

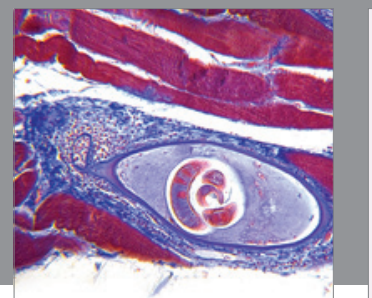

Gastroenterology

Research and Practice
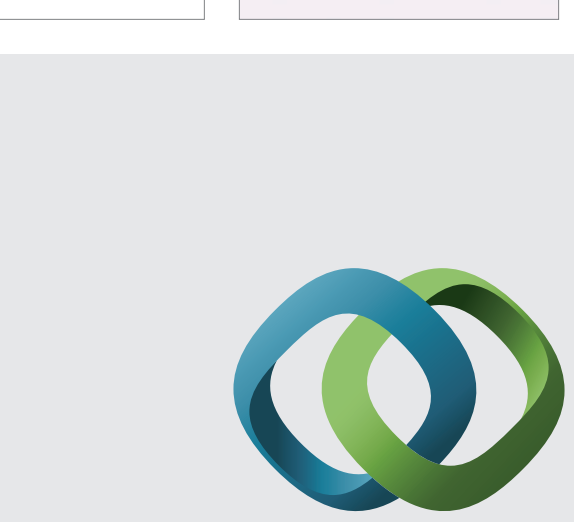

\section{Hindawi}

Submit your manuscripts at

http://www.hindawi.com
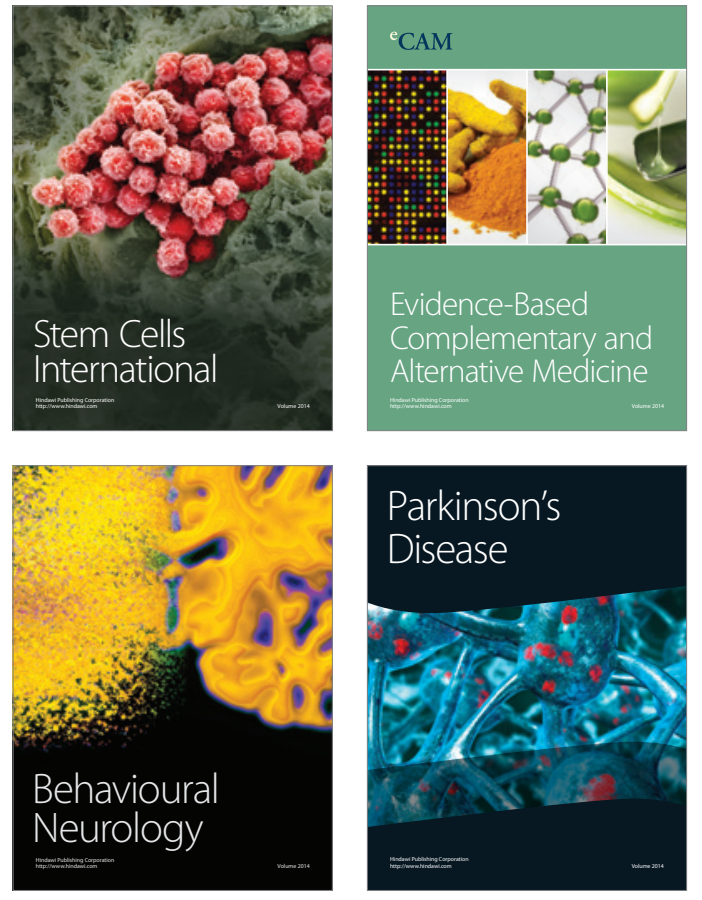
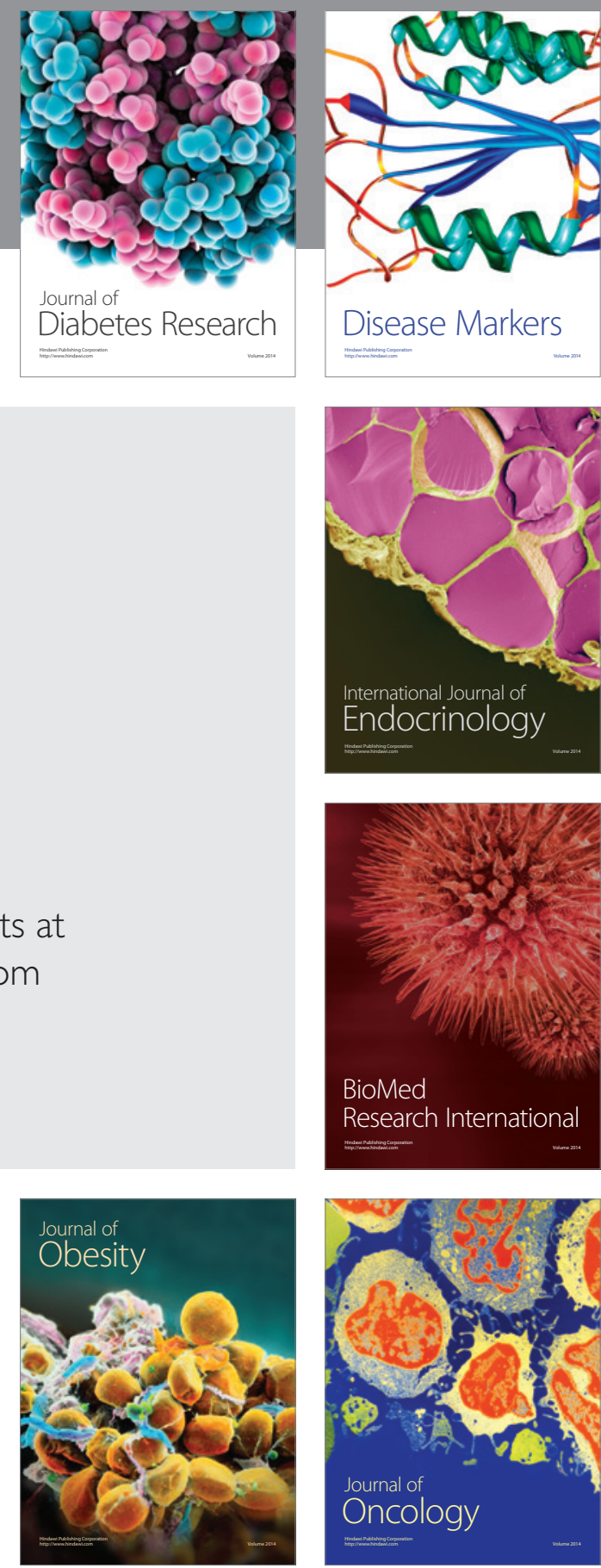

Disease Markers
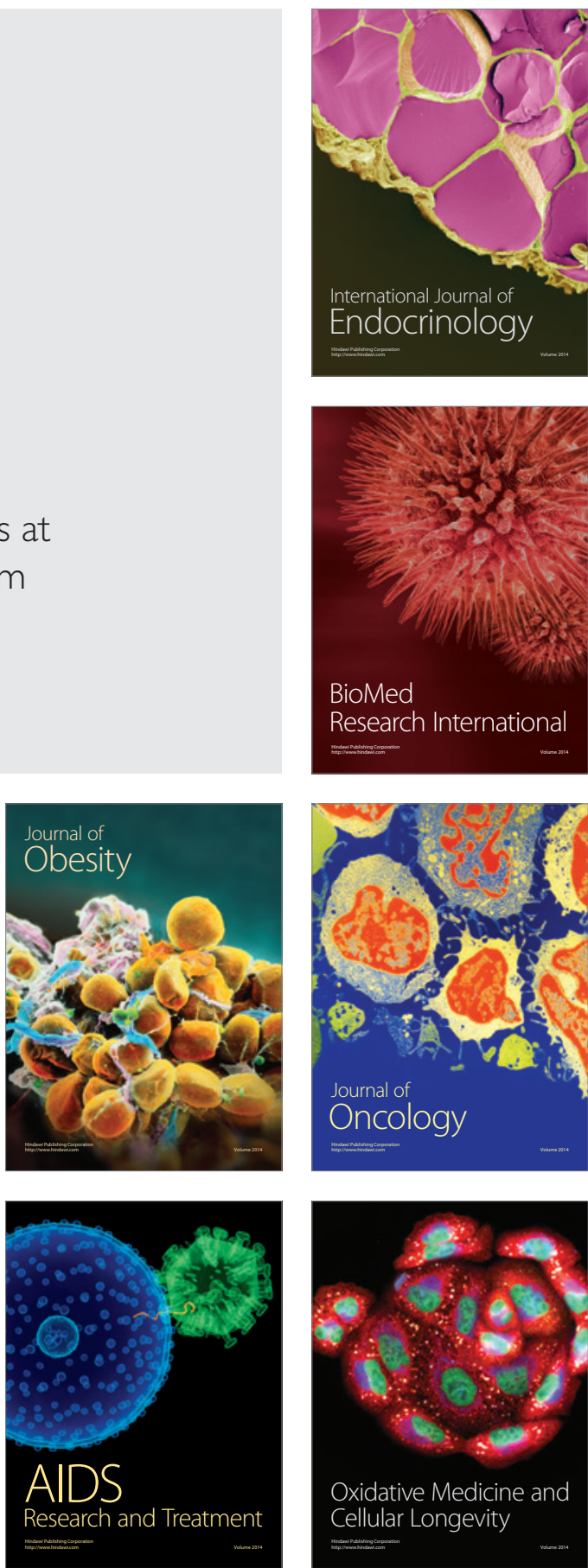